\title{
Article
}

\section{On Metric Invariants of Spherical Harmonics}

\author{
Valentin Lychagin (1) \\ V.A. Trapeznikov Institute of Control Sciences, Russian Academy of Sciences, 65 Profsoyuznaya Str., \\ 117997 Moscow, Russia; valentin.lychagin@uit.no
}

check for

updates

Citation: Lychagin, V. On Metric Invariants of Spherical Harmonics. Symmetry 2021, 13, 1470. https:// doi.org/10.3390/sym13081470

Academic Editors: Anatolij K. Prykarpatski and Stephen Anco

Received: 8 June 2021

Accepted: 2 August 2021

Published: 11 August 2021

Publisher's Note: MDPI stays neutral with regard to jurisdictional claims in published maps and institutional affiliations.

Copyright: (C) 2021 by the author. Licensee MDPI, Basel, Switzerland. This article is an open access article distributed under the terms and conditions of the Creative Commons Attribution (CC BY) license (https:// creativecommons.org/licenses/by/ $4.0 /)$.
Abstract: The algebraic and differential $S O(3)$-invariants of spherical harmonics are studied in this work. The fields of rational algebraic and rational differential invariants and their applications for the description of regular $\mathbf{S O}(3)$-orbits of spherical harmonics are given.

Keywords: differential invariants; spherical harmonics; Euler and Laplace equations

\section{Introduction}

The theory of invariants, or preferably the theory of algebraic invariants, has begun with invariants of binary forms and was developed mainly by pure algebraic methods.

On the other hand, the theory of differential invariants was developed and was used mainly in the geometry of differential equations and as a rule did not use the results of the former theory.

In [1,2], we tried to reconcile these theories and have shown that the correct formulation of the basic theorems in the theory of differential invariants, such as the Lie-Tresse theorem, essentially requires the algebraic theory and, on the other hand, the use of differential invariants in pure algebraic problems could also be successful.

In lectures [3], this idea has been illustrated for the cases of binary and $n$-ary forms.

In this paper, we consider the case of spherical harmonics that are considered as spaces of smooth solutions of Euler and Laplace equations. These equations, as well as spaces of spherical harmonics, possess $\mathbf{S O}(3)$-symmetry.

Moreover, the spherical harmonics, as opposed to the binary forms, play an important role in the theory of differential equations, mathematical physics and especially in applications to Laplace, Poisson and Schrodinger equations [4], as well as in representation theory, where they give the realizations of irreducible $\mathbf{S O}(3)$-representations [5].

In addition, the case of spherical harmonics gives us an excellent opportunity to use and illustrate both invariant theories as well as the basic Rosenlicht and Lie-Tresse theorems.

The paper is organized in the following way. At the beginning, we present the main constructions and results for spherical harmonics. We eliminate the use of the integration procedures that are so common in the theory of spherical harmonics (see, for example [5]) and substitute them with pure algebraic operations. This allows us to apply them also to studies of differential invariants.

We also restricted ourselves to three-dimensional spherical harmonics. This makes our presentation shorter and more transparent, and this case is more important for applications. The generalization of the results of this paper to higher dimensions is straightforward.

\section{Euler-Laplace Equations and Spherical Harmonics}

Spherical harmonics of degree $k$, by definition, are homogeneous polynomials of degree $k$ that are solutions of the Laplace equation (see, for example, $[5,6]$ ).

We denote by $\mathbf{J}^{l}$ the space of $l$-jets of smooth functions on $\mathbb{R}^{3}$ with canonical coordinates $\left(x, y, z, u, u_{x}, u_{y}, u_{z}, u_{x x}, u_{x y}, u_{x z}, \ldots, u_{\sigma}, \ldots\right)$, where $\sigma=\left(\sigma_{1}, \sigma_{2}, \sigma_{3}\right)$ are multi-indices of lengths $|\sigma|=\sigma_{1}+\sigma_{2}+\sigma_{3} \leq l$, and let 


$$
\mathcal{E}_{L}=F_{L}^{-1}(0) \subset \mathbf{J}^{2},
$$

be the Laplace equation, where $F_{L}=u_{x x}+u_{y y}+u_{z z}$, and let

$$
\mathcal{E}_{E}=F_{E}^{-1}(0) \subset \mathbf{J}^{1}
$$

be the Euler equation, where $F_{E}=x u_{x}+y u_{y}+z u_{z}-k u$.

We call the Euler-Laplace equation the chain of algebraic submanifolds

$$
\mathcal{E}_{E L}^{(i)} \subset \mathbf{J}^{i},
$$

given by equations

$$
\mathcal{E}_{E L}^{(1)}=\mathcal{E}_{E}, \mathcal{E}_{E L}^{(i)}=\left\{D^{\alpha}\left(F_{E}\right)=0, D^{\beta}\left(F_{L}\right)=0\right\}, i=2,3, \ldots, k,
$$

where $\alpha=\left(\alpha_{1}, \alpha_{2}, \alpha_{3}\right)$ and $\beta=\left(\beta_{1}, \beta_{2}, \beta_{3}\right)$ are multi-indices of lengths $0 \leq|\alpha| \leq i-1,0 \leq$ $|\beta| \leq i-2, i \geq 2$, and

$$
\mathcal{E}_{E L}^{(k+j)}=\left\{D^{\alpha}\left(F_{E}\right)=0, D^{\beta}\left(F_{L}\right)=0, u_{\sigma}=0\right\}
$$

for $j \geq 1,|\alpha| \leq k-1,|\beta| \leq k-2, k+1 \leq|\sigma| \leq k+j$.

Here

$$
D^{\sigma}=\frac{d^{|\sigma|}}{d x^{\sigma_{1}} d y^{\sigma_{2}} d z^{\sigma_{3}}}
$$

are total derivatives of order $|\sigma|$.

It is easy to check that $\mathcal{E}_{E L}^{(i)}$ are affine manifolds and the natural projections $\pi_{i, i-1}$ : $\mathcal{E}_{E L}^{(i)} \rightarrow \mathcal{E}_{E L}^{(i-1)}$ are affine bundles of dimension 2, for $2 \leq i \leq k$, and $\operatorname{dim} \mathcal{E}_{E L}^{(1)}=6$, (see [7]).

Spherical harmonics of degree $k$ are smooth solutions of the Euler-Laplace equation, and the Lie group $\mathbf{S O}(3)$ of space rotations is the symmetry group of the EulerLaplace equation.

\section{Weyl Algebra and Harmonic Projectors}

We denote by $\mathbb{A}_{3}$ the Weyl algebra on $\mathbb{R}^{3}$ (see, for example [8]), which is the associative algebra of linear differential operators on $\mathbb{R}^{3}$ with polynomial coefficients:

$$
\mathbb{A}_{3}=\left\{\sum_{\sigma} a_{\sigma}(x, y, z) \partial^{\sigma}\right\},
$$

where $a_{\sigma}$ are polynomials (over $\left.\mathbb{R}\right)$ in $(x, y, z)$, and

$$
\partial^{\sigma}=\frac{\partial^{|\sigma|}}{\partial_{x}^{\sigma_{1}} \partial_{y}^{\sigma_{2}} \partial_{z}^{\sigma_{3}}}
$$

The following two Lie subalgebras of $\mathbb{A}_{3}$ are important for our purposes:

1. The Lie algebra $\mathfrak{s l}_{2}(\mathbb{R})$ generated by operators

$$
X_{+}=\frac{1}{2} r^{2}, H=\delta+\frac{3}{2}, X_{-}=\frac{1}{2} \Delta,
$$


where

$$
\begin{aligned}
r^{2} & =x^{2}+y^{2}+z^{2} \\
\delta & =x \partial_{x}+y \partial_{y}+z \partial_{z} \\
\Delta & =\partial_{x}^{2}+\partial_{y}^{2}+\partial_{z}^{2} .
\end{aligned}
$$

Operators $\left(X_{+}, H, X_{-}\right)$form the canonical Weyl basis in $\mathfrak{s l}_{2}$ :

$$
\left[H, X_{+}\right]=2 X_{+},\left[H, X_{-}\right]=-2 X_{-},\left[X_{-}, X_{+}\right]=H .
$$

2. The Lie algebra $\mathfrak{s o}_{3}(\mathbb{R})$ generated by the angular momentum operators

$$
L_{z}=x \partial_{y}-y \partial_{x}, L_{y}=x \partial_{z}-z \partial_{x}, L_{x}=y \partial_{z}-z \partial_{y} .
$$

3. We remark that these Lie algebras mutually commute and form a Howe dual pair [9]. Moreover, the universal enveloping algebra $\mathbf{U}\left(\mathfrak{s l}_{2}\right)$ formed by operators $r^{2}, \delta, \Delta$ is the subalgebra in the Weyl algebra $\mathbb{A}_{3}$ of $\mathfrak{s o}_{3}$-invariant differential operators.

The Casimir or the orbital angular momentum operator for Lie algebra $\mathfrak{s o}_{3}$ has the form

$$
M=L_{x}^{2}+L_{y}^{2}+L_{z}^{2}
$$

or

$$
\begin{aligned}
M= & \left(y^{2}+z^{2}\right) \partial_{x}^{2}+\left(x^{2}+z^{2}\right) \partial_{y}^{2}+\left(x^{2}+y^{2}\right) \partial_{z}^{2}- \\
& 2 x y \partial_{x} \partial_{y}-2 x z \partial_{x} \partial_{z}-2 y z \partial_{y} \partial_{z}-2 \delta .
\end{aligned}
$$

The Casimir operator $M$ could be expressed in terms of operators $r^{2}, \delta, \Delta$. Indeed, we have the following relation:

$$
M=r^{2} \Delta-\delta^{2}-\delta,
$$

for both Casimir operators in $\mathfrak{s o}_{3}$ and $\mathfrak{s l}_{2}$.

Compare this representation with the expression of the Laplace operator $\Delta$ in the spherical coordinates:

$$
\Delta=r^{-2} \partial_{r}\left(r^{2} \partial_{r}\right)+r^{-2} \Delta_{S},
$$

where $\Delta_{S}$ is the spherical Laplace operator; we see that this decomposition could be done in a coordinate-free form, where

$$
\Delta_{S}=M, \partial_{r}\left(r^{2} \partial_{r}\right)=\delta^{2}+\delta .
$$

Let $\mathbb{P}_{l}$ be the space of homogeneous polynomials in $(x, y, z)$ of degree $l$ and $\mathbb{P}_{*}=\oplus_{l} \mathbb{P}_{l}$ be the algebra of polynomials. Then, $\mathbb{P}_{*}$ is a $\mathfrak{s l}_{2}$-module, and its homogeneous components have the following decomposition (see, for example [5]).

Proposition 1. Any homogeneous polynomial $h \in \mathbb{P}_{l}$ could be uniquely presented in the form

$$
h=\sum_{i=0}^{[l / 2]} r^{2 i} h_{l-2 i}
$$

where

$$
h_{l-2 i} \in \mathbb{H}_{l-2 i}=\operatorname{ker}\left(\Delta: \mathbb{P}_{l-2 i} \rightarrow \mathbb{P}_{l-2 i-2}\right)
$$

are homogeneous harmonic polynomials of degree $l-2 i$.

The Howe structure allows us to give the explicit formulae for this decomposition.

To this end, we remark that the action of the Casimir operator $M$ on harmonic polynomials of degree $l$ can be obtained from Formula (1): 


$$
M\left(h_{i}\right)=-i(i+1) h_{i}
$$

Therefore,

$$
M(h)=-\sum_{i=0}^{[l / 2]} r^{2 i}(l-2 i)(l-2 i+1) h_{l-2 i} .
$$

Using this formula, we obtain the following formulae for harmonic projections:

$$
\eta_{l, 2 i}: \mathbb{P}_{l} \rightarrow \mathbb{H}_{l-2 i}, \eta_{l, 2 i}=r^{-2 i} Q_{l, 2 i}(M)
$$

where $Q_{l, 2 i}$ are the polynomials of degree $[l / 2]-1$ and such that

$$
\begin{aligned}
& Q_{l, 2 i}\left(\lambda_{j}\right)=0, j \neq i, \\
& Q_{l, 2 i}\left(\lambda_{i}\right)=1,
\end{aligned}
$$

for $\lambda_{i}=-(l-2 i)(l-2 i+1)$, or explicitly

$$
Q_{l, 2 i}(\lambda)=\prod_{j \neq i}^{[l / 2]} \frac{\lambda-\lambda_{j}}{\lambda_{i}-\lambda_{j}}
$$

We remark also that the exact sequences

$$
\mathbf{0} \rightarrow \mathbb{H}_{l} \rightarrow \mathbb{P}_{l} \stackrel{\Delta}{\rightarrow} \mathbb{P}_{l-2} \rightarrow \mathbf{0}
$$

imply that

$$
\operatorname{dim} \mathbb{H}_{l}=\operatorname{dim} \mathbb{P}_{l}-\operatorname{dim} \mathbb{P}_{l-2}=2 l+1 .
$$

Moreover, the above proposition allows us to identify the harmonic spaces $\mathbb{H}_{l}$ with the quotients $\mathbb{P}_{l} / \mathbb{P}_{l-2}$ by the following exact sequence:

$$
\mathbf{0} \rightarrow \mathbb{P}_{l-2} \stackrel{r^{2}}{\rightarrow} \mathbb{P}_{l} \stackrel{\eta_{k, 0}}{\rightarrow} \mathbb{H}_{l} \rightarrow \mathbf{0}
$$

and introduce the following product structure for harmonic polynomials:

$$
h_{m} * h_{l}=\eta_{m+l, 0}\left(h_{m} h_{l}\right)=\prod_{j=1}^{\left[\frac{m+l}{2}\right]} \frac{M+(m+l-2 j)(m+l-2 j+1)}{2 j(2 j-2 m-2 l-1)}\left(h_{m} h_{l}\right) \text {. }
$$

Example 1. The first harmonic projectors $\eta_{l, 0}$ are of the following form:

$$
\eta_{l, 0}=\prod_{j=1}^{\left[\frac{l}{2}\right]} \frac{M+(l-2 j)(l-2 j+1)}{2 j(2 j-2 l-1)}
$$

and for small degrees, they are

$$
\begin{aligned}
\eta_{2,0} & =-\frac{M}{2 \cdot 3}, \eta_{3,0}=-\frac{M+2}{2 \cdot 5} \\
\eta_{4,0} & =\frac{M(M+6)}{2 \cdot 5}, \eta_{5,0}=\frac{(M+2)(M+12)}{2^{3} 3^{2} \cdot 7}, \\
\eta_{6,0} & =-\frac{M(M+6)(M+20)}{2^{4} 3^{3} \cdot 7 \cdot 11}, \eta_{7,0}=-\frac{(M+2)(M+12)(M+30)}{2^{4} 3^{3} \cdot 11 \cdot 13} .
\end{aligned}
$$


Example 2. We have

$$
\begin{aligned}
& x * y=x y, z * y=z y, x * z=x z \\
& x * x=x^{2}-\frac{r^{2}}{3}, y * y=y^{2}-\frac{r^{2}}{3}, z * z=z^{2}-\frac{r^{2}}{3} .
\end{aligned}
$$

Proposition 2. Spherical harmonics

$$
\mathbb{H}_{*}=\oplus_{l \geq 0} \mathbb{H}_{l}
$$

form a commutative algebra with respect to the product $*$.

This algebra is generated by linear functions $(x, y, z)$ satisfying the relation

$$
x * x+y * y+z * z=0 .
$$

Remark 1. In other words, the complication $\mathbb{H}_{*} \otimes \mathbb{C}$ is the algebra of regular functions on the null cone (6) in $\mathbb{C}^{3}$. It is easy to check that functions $f_{n}(v)=(n, v)^{l}$, where $n \in \mathbb{C}^{3}$ is a vector on the null cone, $(n, n)=0$, and $v \in \mathbb{R}^{3}$, are harmonic functions on $\mathbb{R}^{3}$. Moreover, taking two orthogonal unit vectors $n_{1}, n_{2} \in \mathbf{S}^{2}$, we obtain the harmonic functions

$$
f_{n_{1}, n_{2}}(v)=\left(n_{1}+\sqrt{-1} n_{2}, v\right)^{l}+\left(n_{1}-\sqrt{-1} n_{2}, v\right)^{l} \in \mathbb{H}_{l},
$$

Functions $f_{n}(v)$ generate vector space $\mathbb{H}_{l} \otimes \mathbb{C}$ and functions $f_{n_{1}, n_{2}}(v)$ generate vector space $\mathbb{H}_{l}$.

Example 3. We have the following bases in the spaces of harmonic functions of small degrees:

$$
\begin{aligned}
& \mathbb{H}_{1}=\{x, y, z\}, \mathbb{H}_{2}=\left\{x y, x z, y z, x^{2}-y^{2}, x^{2}-z^{2}\right\} \\
& \mathbb{H}_{3}=\left\{x y z, x^{3}-3 x^{2} y, y^{3}-3 x y^{2}, x^{3}-3 x^{2} z, z^{3}-3 z^{2} x, y^{3}-3 y^{2} z, z^{3}-3 z^{2} y\right\} .
\end{aligned}
$$

Remark 2. Let $L_{n}(x, y, z)=n_{1} x+n_{2} y+n_{3} z$, where $n \in \mathbf{S}^{2}$ is a unit vector. Then, their powers $L_{n}^{l}, n \in \mathbf{S}^{2}$, generate $\mathbb{P}_{l}$, as the vector space, and therefore their $*$-powers

$$
L_{n}^{* l}=L_{n} * \cdots * L_{n}
$$

generate also $\mathbb{H}_{l}$.

Example 4. Let $n=e_{1}=(1,0,0)$; then, $L_{e_{1}}^{* l}=\eta_{k, 0}\left(x^{l}\right)$, and we find from (3) that

$$
\begin{aligned}
L_{e_{1}}^{* 2} & =2 x^{2}-y^{2}-z^{2}, L_{e_{1}}^{* 3}=\frac{x\left(2 x^{2}-3 y^{2}-3 z^{2}\right)}{5}, \\
L_{e_{1}}^{* 4} & =\frac{8 x^{2}\left(x^{2}-3 y^{2}-3 z^{2}\right)+3\left(y^{2}+z^{2}\right)^{2}}{35} .
\end{aligned}
$$

\section{Algebraic and Differential SO(3)-Invariants}

First of all, we remark that the Lie group $\mathbf{S O}(3)$ is algebraic and its actions on algebraic manifolds $\mathcal{E}_{E L}^{(i)}$ by the prolongations are also algebraic.

Therefore, the Hilbert and Rosenlicht theorems are applied to the description of invariants of these actions (see [10]).

Thus, we term polynomial (rational) $\mathbf{S O}(3)$-invariants on $\mathcal{E}_{E L}^{(i)}$ as polynomial (rational) differential invariants of order $i$ for spherical harmonics of degree $k$.

The field of such rational differential invariants is denoted by $\mathcal{F}_{i}^{d}$.

It follows from the structure of the Euler-Laplace equations that the orders of such invariants are not more then the degree $k$ of spherical harmonics under consideration. 
It is also easy to see that $\operatorname{dim} \mathcal{E}_{E L}^{(i)}=2 i+4$, for $i \leq k$, and the regular $\mathbf{S O}(3)$-orbits in $\mathcal{E}_{E L}^{(i)}$ have three dimensions.

Therefore, the Rosenlicht theorem states that the field $\mathcal{F}_{i}^{d}$ of rational differential $\mathbf{S O}(3)$-invariants has a transcendence degree equal to $\operatorname{dim} \mathcal{E}_{E L}^{(i)}-3=2 i+1$.

The spaces $\mathbb{H}_{k}$ of the harmonic polynomial of degree $k$ are spaces of smooth solutions of the Euler-Laplace equations $\mathcal{E}_{E L}^{(k)}$, and they are also vector spaces, where irreducible SO(3)-representations are realized [5].

Here, we have the regular $\mathbf{S O}(3)$-orbits of dimension 3 , when $k \geq 2$.

We remark that orbits of polynomial $L_{n}^{* k}$ are singular in $\mathbb{H}_{k}$ when $k \geq 2$, and regular for $k=1$ where their orbits are spheres $\mathbf{S}^{2}=\mathbf{S O}(3) / \mathbf{S O}(2)$.

Polynomial (rational) SO(3)-invariants of these actions in $\mathbb{H}_{k}$ are called polynomial (rational) algebraic invariants of spherical harmonics with degree $k$.

As we have seen, $\operatorname{dim} \mathbb{H}_{k}=2 k+1$. Therefore, due to the Rosenlicht theorem, the transcendence degree of the field $\mathcal{F}_{k}^{a}$ of rational algebraic $\mathbf{S O}(3)$-invariants has a transcendence degree $2(k-1)$ when $k \geq 2$ and degree 1 when $k=1$.

We remark also (see [3] for more details) that algebraic invariants are also special types of differential invariants.

The Hilbert theorem states that both quotients $\mathbb{H}_{k} / \mathbf{S O}(3)$ and $\mathcal{E}_{E L}^{(k)} / \mathbf{S O}(3)$ are affine manifolds of dimensions $2(k-1)$ and $2 k+1$ respectively, and the fields $\mathcal{F}_{k}^{a}, \mathcal{F}_{k}^{d}$ are the fields of rational functions on them that separate regular orbits (or smooth points).

Therefore, in order to define a regular $\mathbf{S O}(3)$-orbit in $\mathbb{H}_{k}, k \geq 2$, we need $2(k-1)$ rational algebraic $\mathbf{S O}(3)$-invariants that are constants on the orbit and algebraically independent of a neighborhood of the orbit.

On the other hand, the Lie-Tresse theorem [2] states that the orbits are solutions of the quotient differential equations.

In our case, we take a regular harmonic $h \in \mathbb{H}_{k}$. Then, the $\mathbf{S O}(3)$-orbit of the second jet $j_{2}(h) \subset \mathcal{E}_{E L}^{(2)}$ is a 6-dimensional submanifold in the 8-dimensional manifold $\mathcal{E}_{E L}^{(2)}$.

Therefore, we need two independent rational differential invariants of order two to define the orbit. We remark that, in this method for the description of regular orbits, we need only differential invariants of the order two.

\section{Invariant Frames and Coframes}

We start with the observation that functions $J_{-1}=\frac{r^{2}}{2}$ and $J_{0}=u$ are obviously differential SO(3)-invariants.

Therefore, their differential

$$
\omega_{1}=d J_{-1}=x d x+y d y+z d z,
$$

and total differential

$$
\omega_{2}=\widehat{d J}_{0}=u_{x} d x+u_{y} d y+u_{z} d z
$$

are $\mathbf{S O}(3)$-invariant horizontal differential forms.

We take

$$
\omega_{3}=\left(y u_{z}-z u_{y}\right) d x+\left(z u_{x}-x u_{z}\right) d y+\left(x u_{y}-y u_{x}\right) d z .
$$

to be the cross product of 1 -forms $\omega_{1}$ and $\omega_{2}$.

The, $\mathrm{n}$ coframe $\left(\omega_{1}, \omega_{2}, \omega_{3}\right)$ is $\mathbf{S O}(3)$-invariant by the construction. 
Together with invariant coframe $\left(\omega_{1}, \omega_{2}, \omega_{3}\right)$ we obtain the dual (by the metric in $\left.\mathbb{R}^{3}\right)$ frame given by $\mathbf{S O}(3)$-invariant total differentiations:

$$
\begin{aligned}
& D_{1}=x \frac{d}{d x}+y \frac{d}{d y}+z \frac{d}{d z} \\
& D_{2}=u_{x} \frac{d}{d x}+u_{y} \frac{d}{d y}+u_{z} \frac{d}{d z} \\
& D_{3}=\left(y u_{z}-z u_{y}\right) \frac{d}{d x}+\left(z u_{x}-x u_{z}\right) \frac{d}{d y}+\left(x u_{y}-y u_{x}\right) \frac{d}{d z}
\end{aligned}
$$

As we have seen, we expect 5 independent rational differential invariant of order $\leq 2$ for spherical harmonics.

Three of them are obvious and have order $\leq 1$ :

$$
J_{-1}=r^{2}, J_{0}=u, J_{1}=D_{2}(u)=u_{x}^{2}+u_{y}^{2}+u_{z}^{2}
$$

and by using the invariant derivations, we obtain one more invariant:

$$
J_{21}=D_{2}\left(J_{1}\right)=2\left(u_{x}^{2} u_{x x}+u_{y}^{2} u_{y y}+u_{z}^{2} u_{z z}\right)+4\left(u_{x} u_{y} u_{x y}+u_{x} u_{z} u_{x z}+u_{y} u_{z} u_{y z}\right),
$$

To find all invariants, we remark (see [3], for more details) that the following symmetric differential $k$-forms

$$
\Theta_{l}=\sum_{i_{1}+i_{2}+i_{3}=l} u_{i_{1}, i_{2}, i_{3}} \frac{d x^{i_{1}}}{i_{1} !} \frac{d y^{i_{2}}}{i_{2} !} \frac{d z^{i_{3}}}{i_{3} !},
$$

are invariants with respect to the group of affine transformations in $\mathbb{R}^{3}$.

We obtain the following relation between coframes:

$$
\left\|\begin{array}{c}
\omega_{1} \\
\omega_{2} \\
\omega_{3}
\end{array}\right\|=\left\|\begin{array}{ccc}
x & y & z \\
u_{x} & u_{y} & u_{z} \\
y u_{z}-z u_{y} & z u_{x}-x u_{z} & x u_{y}-y u_{x}
\end{array}\right\|\|\| \begin{aligned}
& d x \\
& d y \\
& d z
\end{aligned} \|,
$$

and therefore

$$
\begin{aligned}
\kappa d x & =\left(x J_{1}-n u_{x} J_{0}\right) \omega_{1}+\left(J_{-1} u_{x}-n x J_{0}\right) \omega_{2}+\left(y u_{z}-z u_{x}\right) \omega_{3} \\
\kappa d y & =\left(y J_{1}-n u_{y} J_{0}\right) \omega_{1}+\left(J_{-1} u_{y}-n y J_{0}\right) \omega_{2}+\left(z u_{x}-x u_{z}\right) \omega_{3} \\
\kappa d y & =\left(z J_{1}-n u_{z} J_{0}\right) \omega_{1}+\left(J_{-1} u_{z}-n y J_{0}\right) \omega_{2}+\left(x u_{y}-y u_{x}\right) \omega_{3}
\end{aligned}
$$

on $\mathcal{E}_{E L}^{(n)}$, where

$$
\kappa=J_{-1} J_{1}-n^{2} J_{0}^{2} .
$$

Substituting these expressions into formula (7), we get

$$
\Theta_{l}=\sum_{i_{1}+i_{2}+i_{3}=l} T_{i_{1}, i_{2}, i_{3}} \frac{\omega_{1}^{i_{1}}}{i_{1} !} \frac{\omega_{2}^{i_{2}}}{i_{2} !} \frac{\omega_{3}^{i_{3}}}{i_{3} !},
$$

where $T_{i_{1}, i_{2}, i_{3}}$ are rational differential $\mathbf{S O}(3)$-invariants of order $l$.

Moreover, it follows from the construction of forms $\Theta_{l}$ that all rational SO(3)-invariants are rational functions of $J_{-1}, J_{0}, J_{1}, T_{i_{1}, i_{2}, i_{3}}$.

Certainly, there are relations between them. As we have seen, the bundles $\mathcal{E}_{E L}^{(i)} \rightarrow$ $\mathcal{E}_{E L}^{(i-1)}$ are 2-dimensional, therefore, we expect (and need) only 2 extra independent invariants in pure orders $i \geq 2$. 
We denote by $\nabla$ the invariant derivation $D_{2}$ :

$$
\nabla=u_{x} \frac{d}{d x}+u_{y} \frac{d}{d y}+u_{z} \frac{d}{d z} .
$$

Then, on the level $\mathcal{E}_{E L}^{(2)}$, we have the following invariants $J_{-1}, J_{0}, J_{1}=\nabla\left(J_{0}\right), J_{21}=\nabla^{2}\left(J_{0}\right)$, where

$$
J_{21}=2\left(u_{x}^{2} u_{x x}+u_{y}^{2} u_{y y}+u_{z}^{2} u_{z z}\right)+4\left(u_{x} u_{y} u_{x y}+u_{x} u_{z} u_{x z}+u_{y} u_{z} u_{y z}\right),
$$

together with the trace of the square $J_{22}=\operatorname{Tr}\left(\right.$ Hess $\left.^{2}\right)$ and determinant $J_{23}=\operatorname{det}($ Hess $)$,

$$
\begin{aligned}
& J_{2}=u_{x x}^{2}+u_{y y}^{2}+u_{z z}^{2}+2\left(u_{x y}^{2}+u_{x z}^{2}+u_{z z}^{2}\right), \\
& J_{23}=u_{x x} u_{y y} u_{z z}+2 u_{x y} u_{x z} u_{y z}-\left(u_{x y}^{2} u_{z z}+u_{x z}^{2} u_{y y}+u_{y z}^{2} u_{x x}\right),
\end{aligned}
$$

of the Hesse matrix

$$
\text { Hess }=\left\|\begin{array}{lll}
u_{x x} & u_{x y} & u_{x z} \\
u_{x y} & u_{y y} & u_{y z} \\
u_{x z} & u_{y z} & u_{z z}
\end{array}\right\| .
$$

Due to the Rosenlicht theorem, these invariants generate all rational differential SO(3)-invariants of of order $\leq 2$ for spherical harmonics.

Thus, summarizing this discussion, we obtain the following result.

Theorem 1. The field of rational differential $\mathbf{S O}(3)$-invariants of spherical harmonics is generated by invariants

$$
J_{-1}, J_{0}, \nabla\left(J_{0}\right), J_{2}, \nabla^{2}\left(J_{0}\right), \nabla\left(J_{2}\right), \ldots, \nabla^{i-2}\left(J_{2}\right), \nabla^{i}\left(J_{0}\right), \ldots
$$

This field separates regular $\mathbf{S O}(3)$-orbits in the Euler-Laplace equations $\mathcal{E}_{E L}$.

Remark 3. It is worthwhile to note that all of the above invariants are the same for all harmonics, but invariants of harmonics for the given degree $k$ are obtained by restricting them with the corresponding Euler-Laplace equation.

\section{Structures of Differential SO(3)-Invariants}

\subsection{Monoid Structure}

The results below are similar to the results for SL-actions discussed in [3].

Namely, any smooth function $\phi$ on the jet space $\mathbf{J}^{k}$ could be considered as a scalar differential operator $\hat{\phi}$, acting on functions on $\mathbb{R}^{3}$ in the following way:

$$
\widehat{\phi}: f \in C^{\infty}\left(\mathbb{R}^{3}\right) \longmapsto \mathbf{j}_{k}(f)^{*}(\phi) \in C^{\infty}\left(\mathbb{R}^{3}\right),
$$

where $\mathbf{j}_{k}(f): \mathbb{R}^{3} \rightarrow \mathbf{J}^{k}$ is the $k$-jet of $f$.

Proposition 3 (see [3]). A function $\phi$ is a differential SO(3)-invariant of order $k$ if and only if the operator $\widehat{\phi}$ commutes with the $\mathbf{S O}(3)$-action on $\mathbb{R}^{3}$.

Theorem 2. The field $\mathcal{F}_{*}^{d}$ of rational differential $\mathbf{S O}(3)$-invariants is a monoid with respect to composition

$$
\phi, \psi \in \mathcal{F}_{*}^{d} \longmapsto \phi \diamond \psi \in \mathcal{F}_{*}^{d},
$$

where

$$
\widehat{\phi \diamond \psi}=\widehat{\phi} \circ \widehat{\psi} .
$$

The function $\phi=u$ is the unit in the monoid. 
Example 5. Let $\phi_{\Delta}=u_{x x}+u_{y y}+u_{z z}$ be the function on the space of 2-jets that represents the Laplace operator; i.e., $\widehat{\phi_{\Delta}}=\Delta$. Then, the operator $\widetilde{\Delta}: F \rightarrow \phi_{\Delta} \diamond F$ on the space of functions on the jet spaces acts as follows:

$$
\widetilde{\Delta}=\frac{d^{2}}{d x^{2}}+\frac{d^{2}}{d y^{2}}+\frac{d^{2}}{d z^{2}} .
$$

Example 6. In a similar way, we have

$$
\begin{aligned}
\widetilde{M}= & \left(y^{2}+z^{2}\right) \frac{d^{2}}{d x^{2}}+\left(x^{2}+z^{2}\right) \frac{d^{2}}{d y^{2}}+\left(x^{2}+y^{2}\right) \frac{d^{2}}{d z^{2}}- \\
& 2 x y \frac{d^{2}}{d x d y}-2 x z \frac{d^{2}}{d x d z}-2 y z \frac{d^{2}}{d y d z}
\end{aligned}
$$

for the Casimir operator M.

Example 7. The operator

$$
\widetilde{\delta}=x \frac{d}{d x}+y \frac{d}{d y}+z \frac{d}{d z},
$$

for the scaling vector field is used together with projectors

$$
\pi_{N, n}=\prod_{i \neq n}^{N}(\delta-i),
$$

which send polynomials of degree $\leq N$ to their homogeneous component of degree $n$.

Example 8. The harmonic projectors of homogeneous polynomials of degree $k$ on spherical harmon$i$ cs of degree $2 i$ are also differential operators

$$
\eta_{k, 2 i}=r^{-2 i} Q_{k, 2 i}(M) .
$$

\subsection{Differential Algebra Structure}

Using the metric on $\mathbb{R}^{3}$, we convert (or quantize in the sense of [11]) the symmetric differential forms $\Theta_{k}$ into total differential operators of order $l$ :

$$
\widehat{\Theta}_{l}=\sum_{i_{1}+i_{2}+i_{3}=l} \frac{u_{i_{1}, i_{2}, i_{3}}}{i_{1} ! i_{2} ! i_{3} !} \frac{d^{i_{1}}}{d x^{i_{2}}} \frac{d^{i_{2}}}{d y^{i_{1}}} \frac{d^{i_{3}}}{d z^{i_{3}}},
$$

These operators are $\mathbf{S O}(3)$-invariant and increase the order of differential $\mathbf{S O}(3)$ invariants on $l$ units. Thus, functions $\widehat{\Theta}_{l}(I)$ are differential SO(3)-invariants of order $\leq i+l$ if $I$ is a differential $\mathbf{S O}(3)$-invariant of order $\leq i$.

Example 9. We have

$$
\begin{aligned}
& \widehat{\Theta}_{1}=u_{x} \frac{d}{d x}+u_{y} \frac{d}{d y}+u_{z} \frac{d}{d z}, \\
& \widehat{\Theta}_{2}=\frac{1}{2}\left(u_{x x} \frac{d^{2}}{d x^{2}}+u_{y y} \frac{d^{2}}{d y^{2}}+u_{z z} \frac{d^{2}}{d z^{2}}\right)+u_{x y} \frac{d^{2}}{d x d y}+u_{x z} \frac{d^{2}}{d x d z}+u_{y z} \frac{d^{2}}{d y d z},
\end{aligned}
$$

for first values of $l$.

Applying them to the invariant $J_{0}=u$, we get two invariants:

$$
\widehat{\Theta}_{1}(u)=J_{1}, \widehat{\Theta}_{2}(u)=\frac{J_{22}}{4} .
$$


We remark also that the invariants

$$
G_{k}=\widehat{\Theta}_{k}(u)=\sum_{i_{1}+i_{2}+i_{3}=k} \frac{u_{i_{1}}^{2} i_{2}, i_{3}}{i_{1} ! i_{2} ! i_{3} !},
$$

are proportional to the lengths of the symmetric tensors $\Theta_{k}$.

\subsection{Weights and Algebraic Invariants}

Let

$$
W:=x \partial_{x}+y \partial_{y}+z \partial_{z}+k u \partial_{u}
$$

be the scaling vector field and $W^{*}$ be its $\infty$-prolongation to jet spaces.

Here, as above, $k \in \mathbb{N}$ is the fixed degree of harmonic polynomials under consideration.

We say that a polynomial differential $\mathbf{S O}(3)$-invariant $I$ has weight $w(I) \in \mathbb{N}$ if

$$
W^{*}(I)=w(I) I .
$$

In other words, this means that the value $I(p)$ at a homogeneous polynomial $p$ of degree $k$ is a homogeneous polynomial of degree $w$.

Example 10. We have $w\left(G_{1}\right)=2(k-1), w\left(G_{2}\right)=2(k-2)$ and in general $w\left(G_{l}\right)=2(k-l)$, for $l \leq k$, and $w\left(G_{l}\right)=0$, for $l>k$. We have also $w\left(J_{0}\right)=k, w\left(J_{23}\right)=3(k-2)$.

Finally, we show how to use polynomial differential invariants $G_{l}$ in order to obtain algebraic invariants.

Let $I$ be a differential polynomial $\mathbf{S O}(3)$-invariant of weight $w$ and let $h$ be a harmonic polynomial of degree $k$.

Then, the value $I(h)$ is a polynomial of degree $w$. Applying projectors $\widetilde{\pi_{w, l} l}$, we obtain the new invariants $\widetilde{\pi_{w, l}}(I)$ such that $\widetilde{\pi_{w, l}}(I)(h)$ are homogeneous polynomials of degree $l$ and therefore values of $\mathbf{S O}(3)$-invariants

$$
\widetilde{\eta_{l, 2 s}} \circ \widetilde{\pi_{w, l}}(I)
$$

on harmonic polynomials of degree $k$ are harmonic polynomials of degree $l-2 s$.

Finally, applying invariants $G_{l-2 s}$, we obtain algebraic invariants for all admissible values.

Theorem 3. Let I be a differential $\mathbf{S O}$ (3)-invariant of weight w; then,

$$
\widetilde{G_{l-2 s}} \circ \widetilde{\eta_{l, 2 s}} \circ \widetilde{\pi_{w, l}}(I)
$$

are algebraic invariants for all $w \geq l \geq 2 s$.

Example 11. For harmonics of degree 2, we have algebraic invariants $J_{2}$ and $J_{23}$. Their values on spherical harmonics

$$
h=\lambda_{1} x y+\lambda_{2} x z+\lambda_{3} y z+\lambda_{4}\left(x^{2}-y^{2}\right)+\lambda_{5}\left(x^{2}-z^{2}\right)
$$

are the following:

$$
\begin{aligned}
J_{2}(h) & =2 \lambda_{4} \lambda_{5}+2 \sum_{i=1}^{5} \lambda_{i}^{2} \\
J_{23}(h) & =2 \lambda_{1} \lambda_{2} \lambda_{3}+\lambda_{1}^{2}\left(\lambda_{4}+\lambda_{5}\right)+\lambda_{2}^{2} \lambda_{5}-\lambda_{3}^{2} \lambda_{4}-\lambda_{4}^{2} \lambda_{5}-\lambda_{5}^{2} \lambda_{4} .
\end{aligned}
$$

Funding: The author is partially supported by the RSF grant 21-71-20034.

Conflicts of Interest: The author declares no conflict of interest. 


\section{References}

1. Bibikov, P.; Lychagin, V. Invariants of algebraic group actions from differential point of view. J. Geom. Phys. 2019, 136, 89-96. [CrossRef]

2. Kruglikov, B.; Lychagin, V. Global Lie-Tresse theorem. Sel. Math. New Ser. 2016, 22, 1357-1411. [CrossRef]

3. Lychagin, V.; Roop, M. Differential Invariants in Algebra. arXiv 2021, arXiv:2104.02376.

4. Condon, E.U.; Shortley, G.H. The Theory of Atomic Spectra; Cambridge University Press: Cambridge, UK, 1970.

5. Vilenkin, N.J. Special Functions and the Theory of Group Representations; American Mathematical Society: Providence, RI, USA, 1968.

6. MacRobert, T.M. Spherical Harmonics: An Elementary Treatise on Harmonic Functions, with Applications; Pergamon Press: Oxford, UK, 1967.

7. Krasilshchik, I.S.; Lychagin, V.V.; Vinogradov, A.M. Geometry of jet spaces and nonlinear partial differential equations. In Advanced Studies in Contemporary Mathematics; Sosinskiı̌, A.B., Translator; Gordon and Breach Science Publishers: New York, NY, USA, 1986; Volume 1, p. xx+441.

8. Dixmier, J. Sur les algebres de Weyl. Bull. Soc. Math. Fr. 1968, 96, 209-247. [CrossRef]

9. Howe, R. Remarks on classical invariant theory. Trans. Amer. Math. Soc. 1989, 313, 539-570. [CrossRef]

10. Popov, V.L.; Vinberg, E.B. Invariant Theory. Algebraic Geometry IV. Encyclopaedia of Mathematical Sciences; Springer: Berlin/Heidelberg, Germany, 1994; Volume 55, pp. 123-278.

11. Lychagin, V.; Yumaguzhin, V. On structure of linear differential operators, acting on line bundles. J. Geom. Phys. 2020, 148, 103549. [CrossRef] 